\title{
On the direct evaluation of the equilibrium distribution of clusters by simulation. II
}

\author{
Isamu Kusaka \\ The Koffolt Laboratories, The Department of Chemical Engineering, The Ohio State University, \\ Columbus, Ohio 43210 \\ David W. Oxtoby ${ }^{\text {a) }}$ \\ The James Franck Institute, The University of Chicago, Chicago, Illinois 60637 \\ Zhen-Gang Wang \\ Division of Chemistry and Chemical Engineering, Mail Code 210-41, California Institute of Technology, \\ Pasadena, California 91125
}

(Received 1 May 2001; accepted 7 August 2001)

\begin{abstract}
We clarify some of the subtle issues surrounding the observational cluster method, a simulation technique for studying nucleation. The validity of the method is reaffirmed here. The condition of the compact cluster limit is quantified and its implications are elucidated in terms of the correct enumeration of configuration space. (C) 2001 American Institute of Physics.
\end{abstract}

[DOI: $10.1063 / 1.1406977]$

\section{INTRODUCTION}

Some time ago, we presented a new methodology designed to study nucleation by computer simulation. ${ }^{1-3}$ Though the method was originally intended for vapor to liquid nucleation, it was later shown to be applicable in more general contexts including cavitation $^{4}$ and micelle formation. ${ }^{5}$ However, certain aspects of this observational cluster method have been misunderstood and confusion persists in the literature. For example, Reiss and Bowles 6,7 claimed that the expression we derived for the equilibrium cluster size distribution was in error. Subsequently, we showed that the method they analyzed and criticized was different from the original method we proposed. ${ }^{3}$ Nonetheless, they have maintained their criticism ${ }^{8-10}$ and the misconception has persisted. This has motivated us to elaborate further on some of the subtle issues surrounding our method that might have escaped the attention of others in the field.

\section{CHOICE OF THE COORDINATE SYSTEM IN THE COMPACT CLUSTER LIMIT}

In the present paper, we focus on the method developed for the compact cluster limit (CCL). Roughly speaking, this limit implies that the average intermolecular separation of the condensing molecules in the metastable phase is considerably larger than the physical dimension of the cluster. A precise criterion of CCL is given in Sec. IV C. We note that compactness does not exclude possible openness of the structure of a cluster.

To relate the equilibrium cluster size distributions in a bulk metastable phase to a probability distribution determined for a microscopic subvolume used in a simulation, we employed a fixed molecule frame (FMF), whose origin, taken at the center of the subvolume, coincides with the po-

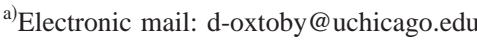

sition of a molecule chosen to identify the position of the cluster. $^{1,3}$ Reference 7 argued that the use of FMF results in an incorrect enumeration of configuration space and that one has to work with the center-of-mass frame (CMF).

By way of illustration, Ref. 7 introduced a model that consists of a dimer of ideal gas molecules confined in one dimension, the analysis of which appeared to indicate that the value of the partition function indeed depended on the choice of the coordinate system. Such a conclusion would be quite astonishing since the partition function, once it is written down, is a multidimensional integral whose value can be evaluated in any coordinate system. In fact, Ref. 3 pointed out that the limits of integration were not properly transformed in Ref. 7.

The real issue, however, lies elsewhere. That the system boundary must be transformed indicates that the physical properties of the cluster are sensitive to it. Moreover, the transformation of the limits of integration is not trivial for clusters larger than a dimer. Thus, if a cluster were to be characterized by its size alone and one were to adhere to simple boundary conditions, the physical properties of the cluster measured under different observational situations (specified in this case by the choice of the coordinate system and the system boundary) could not be related to each other in any simple fashion, and in that sense the cluster would lose its objective attributes. A similar remark applies when the system size is changed without changing the coordinate system. The key observation is that when the properties of the cluster depend sensitively on the system boundary, the very concept of cluster that is characterized by its size alone is no longer appropriate as a tool to describe the objective reality of the nucleation process. In the wording of Ref. 1, our coarse-grained description of nucleation loses its "focus" since, for example, a configuration more properly regarded as a vapor molecule plus an $N$-sized cluster is counted as a configuration of an $(N+1)$-sized cluster. The 
ideal gas model is one such example. The apparent difficulty one encounters for this model is simply a reflection that the model lies outside CCL.

Within CCL, the system boundary can be taken to be considerably larger than the physical dimension of the cluster. Agreement is thus reached among simulations carried out with different choices of coordinate system, without accounting for the transformation of the limits of integration. ${ }^{3} \mathrm{~A}$ recent simulation study by $\mathrm{Oh}$ and $\mathrm{Zeng}^{11}$ is quite reassuring in this respect. They performed a simulation for a binary mixture of SPC/E water and Lennard-Jones particles and found no noticeable difference in the cluster size distributions obtained using FMF and CMF.

In a recent paper, Reiss and Bowles (RB) analyzed a one-dimensional ideal gas trimer and argued that FMF undercounts the relevant configurations. ${ }^{10}$ Once again, their conclusion results from the simple fact that the model lies outside CCL. Nonetheless, a closer analysis of the model provides deeper insight into the significance of this limit.

In our method, the partition function $Q_{3}$ for the trimer is constructed by fixing molecule 1 at the center of the system of length $2 h$, while the other two molecules can be anywhere in the system. This results in

$$
Q_{3}=\frac{1}{3 ! \Lambda^{3}} \int_{0}^{L} d x_{1} \int_{-h}^{h} d \xi_{21} \int_{-h}^{h} d \xi_{31}=\frac{2 L h^{2}}{3 \Lambda^{3}},
$$

where $\Lambda$ is the thermal wavelength, $x_{i}$ denotes the position of molecule $i$, and $\xi_{i j}=x_{i}-x_{j}$. The partition function they compared with $Q_{3}$ can be written as

$$
Q_{3}^{\mathrm{RB}}=\frac{1}{3 ! \Lambda^{3}} \sum \int_{0}^{L} d x_{1} \int_{-h}^{0} d \xi_{21} \int_{0}^{h} d \xi_{31}=\frac{L h^{2}}{\Lambda^{3}},
$$

where the multidimensional integral in Eq. (2) is obtained under the constraints that $x_{2} \leqslant x_{1} \leqslant x_{3}$ and that molecules 2 and 3 stay within the distance $h$ from molecule 1 . The sum is taken over all possible permutations of the particle labels. Equation (2) was thus obtained using a criterion that the three molecules constitute a trimer if they are confined within the distance $h$ from the central molecule, while allowing any one of the molecules to be the central one. Equations (1) and (2) disagree because they are constructed for different entities. Under the physically relevant situations where the Boltzmann factor brings the system to CCL, the disagreement disappears.

The point can be best illustrated by showing that the two constructions of the partition function result in the identical result when the maximum intermolecular distance is constrained to be less than $h$. Our approach results in

$$
\begin{aligned}
Q_{c 3}= & \frac{1}{3 ! \Lambda^{3}} \int_{0}^{L} d x_{1}\left(\int_{-h}^{0} d \xi_{21} \int_{-h}^{\xi_{21}+h} d \xi_{31}\right. \\
& \left.+\int_{0}^{h} d \xi_{21} \int_{\xi_{21}-h}^{h} d \xi_{31}\right)=\frac{L h^{2}}{2 \Lambda^{3}} .
\end{aligned}
$$

The construction that led to Eq. (2), when supplemented with this additional constraint, yields the same value as Eq. (3). [See Eq. (17) of Ref. 10.]
One might argue for $Q_{3}^{\mathrm{RB}}$ on the basis of its symmetric nature. However, the usefulness of the partition function is limited to CCL, since its value depends sensitively on the choice of system size outside CCL. A similar observation applies when comparing the LBA cluster ${ }^{12}$ and the Stillinger cluster. ${ }^{13}$ Outside CCL, their physical properties do not agree and are also sensitive to the parameters defining them, quite irrespective of the fact that both of them employ CMF. This is exactly the difficulty which had prevented the development of a molecular simulation approach to nucleation. Simply adopting CMF does not resolve the difficulty.

\section{PROBABILITY OF CLUSTER LOCATION WITHIN A SUBVOLUME}

Our use of FMF was further criticized in Ref. 10 on the basis of a claim that the probability distribution of the central molecule over the subvolume $V$ is nonuniform. However, the probability Ref. 10 focused on differs from what we used and the criticism bears no relevance to our method.

According to Ref. 10, the probability $\chi(\lambda \mid L)$ that, given the existence of the one-dimensional ideal gas dimer (defined as two molecules within a distance $h$ from each other) somewhere in $L$, it will be found in $\lambda$ is

$$
\begin{aligned}
& \chi(\lambda \mid L)=\frac{\lambda}{L}\left(1+\frac{h}{2 \lambda}\right) \quad(\text { if } \lambda>h), \\
& \chi(\lambda \mid L)=\frac{\lambda}{L}\left(2-\frac{\lambda}{2 h}\right) \quad(\text { if } \lambda<h) . \quad[\text { Ref. 10]. }
\end{aligned}
$$

When multiplied by the factor $L / \lambda$, i.e., the number of cells of length $\lambda$, the probability $\chi$ is observed not to be properly normalized, both expressions being larger than unity. This rather unsettling result follows from the fact that, in calculating $\chi$, configurations are included in which molecule 1 lies outside $\lambda$. One such situation is represented by Fig. 4(c) of Ref. 10 , contributing $h^{2} / 2$ to the partition function $I_{b}$. [See Eq. (23) of Ref. 10.] The other situation is in their Fig. 4(d) with labels between molecules exchanged, resulting in the partition function $I_{a}^{\prime}=h^{2} / 2$. [See Eq. (24) of Ref. 10.] The same is true with molecule 2 being outside $\lambda$. In Ref. 10, therefore, the location of the dimer is identified sometimes with that of molecule 1 and the other times with that of molecule 2. As a result, when $\chi$ is calculated for adjacent cells, its value must be smaller than that given by Eq. (4), leading to the alleged nonuniformity in the probability distribution. If this nonuniformity is ignored, redundancy would be introduced in enumerating the configurations, leading to the observation that $\chi$ is not properly normalized. Evidently, the nonuniformity depends on the choice of $\lambda$. The apparent contradiction with the underlying symmetry of space, however, is an artifact of their particular method of identifying the location of a cluster, which is different from what we proposed.

In our approach, the location of the cluster is identified with that of molecule 1 only. In this case, the partition function of the dimer obtained under the restriction that the cluster lies inside $\lambda$ is given by 


$$
Q_{2}^{\lambda}=\frac{1}{2 ! \Lambda^{2}} \int_{0}^{\lambda} d x_{1} \int_{-h}^{h} d \xi_{21} .
$$

When the dimer can be anywhere in $L$, the partition function becomes

$$
Q_{2}^{L}=\frac{1}{2 ! \Lambda^{2}} \int_{0}^{L} d x_{1} \int_{-h}^{h} d \xi_{21} .
$$

The ratio between these two partition functions yields the desired probability

$$
\chi(\lambda \mid L)=\frac{\lambda}{L},
$$

which is properly normalized and manifestly consistent with the symmetry of space.

Equation (4) was obtained by Reiss and Bowles by treating the different parts of space differently. The so-called mapping process, i.e., construction of the equilibrium cluster size distribution, is extremely complicated in this case. In contrast, our method treats different parts of space on the same footing and results in the simplest mapping procedure, as we see in Sec. IV A. A particular molecule can be singled out for the purpose of locating the cluster. This has been demonstrated in Ref. 3 for a one-dimensional ideal gas dimer and in Sec. II for a trimer in CCL. Section IV C validates the procedure for a cluster of arbitrary size and there is no need to adopt the complicated method that leads to Eq. (4).

\section{CLUSTER SIZE DISTRIBUTION IN THE COMPACT CLUSTER LIMIT}

\section{A. Law of mass action}

In this section, we rederive the cluster size distribution for the case of CCL. Though such derivations have been presented by others, ${ }^{14,15}$ the particular derivations they have chosen do not illuminate certain aspects we wish to clarify. Unlike the derivation in Ref. 3, we shall make use of the fact that, in CCL, the vapor phase can be regarded as an ideal gas mixture of clusters of various sizes.

Consider a canonical ensemble of $N_{\text {tot }}$ molecules in volume $V_{\text {tot }}$. Now, imagine that the $N_{\text {tot }}$ molecules can form clusters of sizes $N=1, \ldots, N_{\text {cut }}$ and let $n_{i}$ denote the number of $i$-sized cluster. The partition function of the system is

$$
Q_{\mathrm{tot}}=\sum_{n_{i}=0}^{*} \prod_{i=1}^{N_{\text {cut }}} \frac{q_{i}^{n_{i}}}{n_{i} !}=\prod_{i=1}^{N_{\text {cut }}} \sum_{n_{i}=0}^{*} \frac{q_{i}^{n_{i}}}{n_{i} !},
$$

where $N_{\text {cut }}$ is the maximum size of cluster allowed in the system and is conventionally taken to exceed slightly the size of the critical nucleus to confine the system to a metastable state. The $*$ in the summation accounts for the conservation of molecules

$$
\sum_{i=1}^{N_{\text {cut }}} i n_{i}=N_{\text {tot }} .
$$

In Eq. (8), $q_{i}$ is the partition function of an $i$-sized cluster, including the overall translational and the internal degrees of freedom of the cluster; its evaluation will be discussed later. A common procedure to circumvent the inconvenience due to Eq. (9) is to introduce a Lagrange multiplier and resort to the maximum term approximation, under which the sum of the product term is replaced by the maximum product term. ${ }^{14}$ Alternatively, we work with the grand canonical ensemble, the relevant partition function for which is

$$
\Xi_{\mathrm{tot}}=\prod_{i=1}^{N_{\text {cut }}} \sum_{n_{i}=0}^{\infty} \frac{q_{i}^{n_{i}}}{n_{i} !} \exp \left(\beta \mu_{i} n_{i}\right)=\exp \left[\sum_{i=1}^{N_{\text {cut }}} q_{i} e^{\beta \mu_{i}}\right],
$$

where $\mu_{i}$ is a fictitious $i$-dependent chemical potential of an $i$-sized cluster. From Eq. (10), the average number of the $\mathrm{N}$-sized clusters is

$$
\left\langle n_{N}\right\rangle=\frac{\partial \ln \Xi_{\text {tot }}}{\partial \beta \mu_{N}}=q_{N} e^{\beta \mu_{N}} .
$$

A physically relevant result is obtained by setting $\mu_{i}=i \mu$, where $\mu$ is the chemical potential of monomer in the vapor phase. Thus, the cluster size distribution is found to be

$$
c(N) \equiv \frac{\left\langle n_{N}\right\rangle}{V_{\text {tot }}}=\frac{1}{V_{\text {tot }}} q_{N} e^{\beta N \mu} .
$$

The above-mentioned maximum term approximation yields the same result.

The cluster partition function $q_{N}$ can be written as

$$
q_{N}=\frac{1}{N ! \Lambda^{3 N}} \int_{c} d \mathbf{r}^{N} e^{-\beta U_{N}} .
$$

The subscript $c$ indicates that the $N$ molecules have to be in a cluster. This fact is reflected by constraining the $N$ molecules to be in a small volume $V$ around the center of mass of the cluster, or more conveniently, around a tagged molecule 1. Using the latter, i.e., by transforming the coordinates from the laboratory system to FMF, we may write $q_{N}$ as

$$
q_{N}=\frac{1}{N ! \Lambda^{3 N}} \int_{V_{\mathrm{tot}}} d \mathbf{r}_{1} \int_{V}^{\odot} d \mathbf{r}^{N-1} e^{-\beta U_{N}},
$$

where the second configuration integral is taken over $V$ with molecule 1 fixed at its center as indicated by the superscript $\odot . \mathbf{r}^{N-1}$ collectively denotes the coordinates of the remaining $N-1$ molecules.

In writing Eq. (14), a particular choice is made for the integration limit, which was previously specified by the subscript $c$ in Eq. (13). Section IV C provides a rigorous proof that Eq. (14) correctly exhausts the configuration space, thereby validating the particular choice we have adopted here.

In the absence of any external field, $U_{N}$ and hence the second integral in Eq. (14) are independent of $\mathbf{r}_{1}$. Thus, $q_{N}$ can be written as

$$
q_{N}=\frac{1}{N ! \Lambda^{3 N}} \frac{V_{\text {tot }}}{V} \int_{V} d \mathbf{r}_{1} \int_{V}^{\odot} d \mathbf{r}^{N-1} e^{-\beta U_{N}} .
$$

The integration over $\mathbf{r}_{1}$ was previously referred to as the analytical integration carried out while deliberately ignoring the interaction between the cluster and the system boundary. ${ }^{1,3}$

In terms of $\Xi$ and $p_{c}$, defined, respectively, by 


$$
\Xi \equiv \sum_{N=0}^{N_{\text {cut }}} \frac{z^{N}}{N !} \int_{V} d \mathbf{r}_{1} \int_{V}^{\odot} d \mathbf{r}^{N-1} e^{-\beta U_{N}},
$$

and

$$
p_{c}(N) \equiv \frac{1}{\Xi} \frac{z^{N}}{N !} \int_{V} d \mathbf{r}_{1} \int_{V}^{\odot} d \mathbf{r}^{N-1} e^{-\beta U_{N}},
$$

where $z=\exp (\beta \mu) / \Lambda^{3}$ is the fugacity, Eq. (12) may be written as

$$
c(N)=\frac{1}{V} p_{c}(N),
$$

where we replaced $\Xi$ by unity since the essence of our approach for CCL is to take $V$ sufficiently small so that $z V$ is negligible compared to unity. ${ }^{1}$

The probability $p_{c}$ itself is not convenient to evaluate by simulation. Thus, we introduced $p^{\odot}$ defined by ${ }^{1}$

$$
p^{\odot}(N) \equiv \frac{1}{\Xi^{\odot}} \frac{z^{N}}{N !} \int_{V}^{\odot} d \mathbf{r}^{N-1} e^{-\beta U_{N}},
$$

where

$$
\Xi^{\odot} \equiv \sum_{N=1}^{N_{\max }} \frac{z^{N}}{N !} \int_{V}^{\odot} d \mathbf{r}^{N-1} e^{-\beta U_{N}}
$$

Note that $p^{\odot}$ is normalized for $N=1, \ldots, N_{\max }, N_{\max }$ denoting the maximum size of the cluster being simulated.

The relation between $p_{c}$ and $p^{\odot}$ was derived previously and is given by ${ }^{1,3}$

$$
\begin{aligned}
& p_{c}(0)=\frac{p^{\odot}(1)}{p^{\odot}(1)+z V \sigma} \\
& p_{c}(N)=\frac{z V p^{\odot}(N)}{p^{\odot}(1)+z V \sigma} \quad\left(N=1, \ldots, N_{\text {cut }}\right) \\
& \sigma \equiv \sum_{N=1}^{N_{\text {cut }}} p^{\odot}(N),
\end{aligned}
$$

which was obtained by eliminating $\Xi^{\odot} / \Xi$ from the relations

$$
\begin{aligned}
& p_{c}(1)=z V p_{c}(0) \\
& p_{c}(N)=\frac{\Xi^{\odot} V}{\Xi} p^{\odot}(N) \quad\left(N=1, \ldots, N_{\text {cut }}\right),
\end{aligned}
$$

in favor of the normalization condition of $p_{c}$

$$
\sum_{N=0}^{N_{\text {cut }}} p_{c}(N)=1 .
$$

Equation (22) itself follows from Eqs. (17) and (19).

In the present derivation, Eq. (18) was obtained using standard statistical mechanical machinery. Equation (14) completely determines the physical and mathematical contents of the relevant partition functions. It is clear from Eq. (14) that no interaction exists between the cluster and the system boundary and that the probability distribution for the position of the cluster, when identified with that of molecule 1 , is uniform throughout $V_{\text {tot }}$ since $U_{N}$ depends only on the relative position of molecules. FMF is simply a matter of mathematical convenience in evaluating Eq. (14). We use $p_{c}$ and $p^{\odot}$ since they allow us to avoid the surface effect altogether and carry over the uniformity into a simulation that focuses on $V$ despite the fact that $V$ is comparable to the spatial extent of the cluster itself.

\section{B. Comment on our earlier derivation}

In Ref. 1, Eq. (16) was written simply as

$$
\Xi=\sum_{N=0}^{N_{\text {cut }}} \frac{z^{N}}{N !} \int_{V} d \mathbf{r}^{N} e^{-\beta U_{N}},
$$

where the configuration integral should not be confused with a partition function of $N$ molecules confined entirely to $V$, with which the probability $p^{\otimes}(N)$ as defined in Ref. 3 is associated. In fact, the meaning of this integral has been misinterpreted as such in Refs. 6-10. However, $p^{\otimes}$ reflects significant surface effects due to the interaction between molecules and the container. The very motivation of introducing $p^{\odot}$ was to avoid such a surface effect. ${ }^{1}$ The distinction between $p_{c}$ and $p^{\otimes}$ was also a major focus of Ref. 3 .

The rationale for writing $\Xi$ in the form of Eq. (24) is in our original derivation of Eq. (18). First, Eq. (24) was written as a grand canonical partition function for a macroscopic volume $V$ chosen to satisfy two conditions given in Ref. 1. At this stage, $N$ molecules may be regarded as being confined entirely to $V$ and Eq. (24) is the ordinary partition function. Equation (18) applies in this case because the cells of volume $V$ are statistically independent and the surface effect is negligible for a macroscopic $V$. Then, a limiting process is taken in which $V$ becomes sufficiently small to suppress the vapor contribution. As a result, the observational situation specified by $V$ isolates a single cluster. Since most of the cells are now empty and the interaction among them is sufficiently weak, statistical independence among the cells still applies and the same expression for the cluster size distribution remains valid. The only problem is that the surface effect is no longer negligible for a small $V \cdot p^{\odot}$ is simply a device that allows us to take the indicated limiting process while avoiding the surface effect. As a result, the integration limit in Eq. (24), written for a microscopic $V$, is understood in the sense indicated by Eq. (16).

The line of reasoning just given was preferred in Ref. 1 in order to emphasize a nontrivial aspect of the observational cluster. In Ref. 15, for example, the cluster size distribution was derived using $\Xi$ written for a macroscopic $V$ by assuming the existence of explicit cluster criteria. In contrast, our approach does not require any cluster criteria: the precise characteristics of the cluster reveal themselves with increasing clarity as the vapor contribution is suppressed more effectively by making $V$ smaller.

\section{Enumeration of configuration space in the compact cluster limit}

CCL prevails when nucleation occurs in an attenuated vapor. Since CCL is to some extent an idealized notion, our approach is an approximation that becomes accurate at CCL. It is of interest to examine the nature of the approximations implicit in CCL. Our goal is accomplished most succinctly by resorting to the notion of generic phase space. ${ }^{16}$ In a 
generic phase space, two phase points that are related by mere exchange of identical particles are considered identical, while they are considered distinct in the specific phase space.

Note first that out of $N$ ! configurations that can be obtained by permutation of the labels of molecules, only ( $N$ $-1)$ ! of them are realized in the integral Eq. (19). In this sense, $p^{\odot}$ defies a purely physical interpretation and should be regarded simply as a mathematical convenience that leads to a very simple procedure in enumerating the configuration space.

In fact, provided that the system radius can be taken to be larger than the diameter of the cluster, defined as the largest distance between the molecules in the system, the remaining permutations are seen to occur upon integration with respect to the coordinates of molecule 1 over the whole volume $V_{\text {tot }}$. The extra factor $1 / N$ is included here in anticipation of this integration.

More formally put, for a given generic configuration of a cluster placed somewhere in $V_{\text {tot }}$, each of the $N$ ! specific configurations does occur in the integral

$$
\int_{V_{\mathrm{tot}}} d \mathbf{r}_{1} \int_{V}^{\odot} d \mathbf{r}^{N-1} e^{-\beta U_{N}} .
$$

In fact, under the above-mentioned choice of the system, any specific configuration of the cluster, obtained by arbitrarily labeling the $N$ molecules, can be enclosed by a spherical container centered around molecule 1. Since each specific configuration evidently occurs exactly once in Eq. (25), it follows that for a given generic configuration, exactly $N$ ! specific configurations are included in Eq. (25). On the other hand, for any given specific configuration included in Eq. (25), there exists a generic configuration. Since $U_{N}$ is independent of the labels of the molecules, each of the $N$ ! specific configurations derived from a given generic phase contributes to Eq. (25) to the same extent. It follows that the entire assembly of specific configurations enumerated by Eq. (25) can be divided into sets, each consisting of $N$ ! specific configurations that are equivalent in the generic configuration space and make an identical contribution to Eq. (25). Consequently, Eq. (25), when divided by $N$ !, correctly exhausts the generic configuration space, thereby reconfirming the correctness of our approach.

The above argument presumes that the system radius can be taken to be larger than the diameter of the cluster. This condition is satisfied in CCL, as we shall now see. Consider the partition function

$$
\xi_{N}^{\odot} \equiv \frac{z^{N}}{N !} \int_{V}^{\odot} d \mathbf{r}^{N-1} e^{-\beta U_{N}} .
$$

This partition function includes contributions from configurations in which some of the molecules are more properly regarded as part of the vapor. ${ }^{1}$ A rough estimate for this contribution is obtained by assuming the presence of a monomer decoupled from the rest of the $N-1$ molecules

$$
\xi_{p N}^{\odot} \equiv \frac{z^{N} V}{N !} \int_{V}^{\odot} d \mathbf{r}^{N-2} e^{-\beta U_{N-1}}
$$

Dividing Eq. (27) by Eq. (26), we find the probability of finding a system with one "vapor molecule." The probability can be written as $z_{N} V / N$, where $z_{N}$ is the value of the fugacity at which $\xi_{N}^{\odot}=\xi_{N-1}^{\odot}$ holds true, meaning that the cluster of $N$ molecules, roughly speaking, is a critical nucleus. Since the notion of vapor contribution is relevant only when $N$ $\geqslant 2$, the contribution is negligible if

$$
\frac{z_{N} V}{N} \ll 1,
$$

for $2 \leqslant N \leqslant N_{\text {cut }}$, which quantifies the condition of CCL. The appearance of $z_{N}$ rather than $z$ is reasonable since the configuration integral itself is independent of $z$, while $z_{N}$ is determined once the temperature is specified.

The enumeration of the configuration space by Eq. (25) is correct only when the diameter of the cluster does not exceed the radius of the system. Since we are concerned only with the problematic configurations that cannot be corrected by choosing a larger system size, the problematic configurations have to do with the vapor contribution. Recalling that the basic idea in Ref. 1 was to suppress this contribution, we correct our partition function $\xi_{N}^{\odot}$ by subtracting $\xi_{p N}^{\odot}$, thereby obtaining the correction factor to multiply $\xi_{N}^{\odot}$ as

$$
1-\frac{z_{N} V}{N}
$$

which is essentially unity under CCL.

The correct enumeration of the configuration space proven here, of course, is only necessary but not sufficient for the relevance of the partition function in nucleation. Physically, CCL is required since the cluster cannot be characterized by its molecular content alone outside this limit. A similar remark applies to CMF. There are, however, certain practical considerations in choosing the coordinate system. On the one hand, CMF allows one to work with a smaller system volume, thereby suppressing the vapor contribution more effectively. However, ensuring detailed balance for grand canonical moves may involve some complication. In a naive algorithm, where a molecule is chosen randomly for a trial removal irrespective of its position in the container, detailed balance demands that upon a trial creation of a molecule, its position must be generated uniformly within the spherical container which is centered around the center of mass that would result if the trial creation were accepted. The error committed by not satisfying detailed balance precisely, however, is expected to be negligible in CCL since the problematic situation occurs only when the molecule in question, or the position for the trial creation, is far from the center of mass. On the other hand, a grand canonical move is much simpler to implement in FMF, or alternatively, under periodic boundary conditions.

\section{On the concept of the observational cluster}

Admittedly, the condition that the diameter of the cluster be smaller than the system radius can be formulated as explicit cluster criteria. However, we have taken a different viewpoint.

If a molecular aggregate is placed in vacuum, there is no ambiguity as to what constitutes a cluster, provided that no evaporation takes place. When the same aggregate is placed 
in vapor, it is no longer possible to state in a completely objective manner exactly which molecules belong to the cluster. Conventional theories deal with the problem by introducing explicit cluster definitions.

Recall, however, that our goal is to construct a statistical description of nucleation. In general, this is realized by first specifying the observational situation and then introducing a coarse graining to what we observe. In this context, our observational cluster refers to a macrostate that emerges as a result of coarse graining. When the macrostate is characterized by global order parameters, the question as to which molecules belong to the cluster does not arise. Thus, at least conceptually, the observational cluster is entirely different from what conventional theories have envisioned.

The advantage of our viewpoint is twofold. (1) The method can be easily generalized to address nucleation outside CCL. ${ }^{2,3}$ (2) The method places itself quite naturally on a pathway of successive levels of coarse graining that starts from the absolute molecular level details and leads eventually to the thermodynamics of interface ${ }^{17}$ while passing through other powerful machinery such as the density functional approach. ${ }^{18}$ Not surprisingly, the method provides a unified perspective in understanding a wide variety of nucleation phenomena.

\section{COMPARISON BETWEEN THE TWO METHODOLOGIES}

The existing criticism from the authors of Refs. $6-10$ is largely due to confusion on their part regarding the physical or mathematical contents of the probabilities we use, i.e., $p_{c}$ and $p^{\odot}$. This section clarifies this confusion.

In Ref. 10 three types of clusters were discussed: (1) a type-A cluster consisting of $N$ indistinguishable molecules confined entirely in $V$; (2) a type-B cluster defined as $N$ indistinguishable molecules confined entirely in $V$ with (any) one of $N$ molecules placed at the center; (3) a modified B cluster, which differs from the type-B cluster in that the central molecule is always 1 and distinguishable from the rest of the molecules in $V_{\text {tot }}$. In the present notation, the probability of finding a type A cluster in $V$ is $p^{\otimes}$, while the probabilities of finding a type-B cluster and a modified type-B cluster in $V$ will be denoted by $p^{*}$ and $p^{* *}$, respectively.

Reference 10 put forward the following equation:

$$
p^{\odot}(N)=p^{*}(N)=\frac{1}{N} p^{* *}(N), \quad[\text { Ref. 10] }
$$

based on which, Ref. 10 criticizes Eq. (18) by claiming that the analytical integration mentioned in connection to Eq. (15) assumes uniformity of the relevant probability distribution. As we have shown in Sec. IV, their claim simply is not true. The error in the reasoning of Ref. 10 lies in the misconception that $p^{\odot}$ represents $p^{*}$ because of the $1 / N$ ! factor in Eq. (19). Such an interpretation is incorrect since all of the $N$ molecules in the configuration integral are labeled and the molecule at the origin always carries the label 1 . The additional factor $1 / N$ does not change this fact. Stated differently, $p^{\odot}$ is a mathematical object introduced to facilitate the evaluation of $q_{N}$ as defined in Eq. (14). The issue regarding indistinguishability of identical particles plays no role in the mathematical procedure of evaluating $q_{N}$ or $p^{\odot}$.

As we shall see shortly, the correct equation is

$$
p^{\odot}(N) \approx \frac{1}{N} p^{*}(N)=\frac{1}{N} p^{* *}(N),
$$

and hence their criticism does not apply. The Appendix explores another route to arrive at Eq. (31). The physical meaning of this result is quite obvious. In $p^{\odot}$, there is only one way of choosing the central molecule, while in $p^{*}$, there are $N$ ways of doing so.

The simple relation between $p^{\odot}$ and $p^{*}$ does not apply directly to the two corresponding partition functions $Q_{N}$ and $Q_{N}^{\mathrm{RB}}$ because of the difference in the mapping procedures. See Eqs. (1) and (2), for example. Thus, the fact that $p^{\odot}$ $\neq p^{*}$ does not contradict our earlier conclusion regarding the agreement between these two partition functions in CCL.

The proof of Eq. (31) is quite simple. By eliminating $Q_{N}^{* *} / Q(N, V)$ from Eqs. (A3), (A4), and (A5) of Ref. 10, one finds

$$
p^{* *}(N) \equiv \frac{1}{\Xi * *} \frac{z^{N-1}}{(N-1) !} \int_{V}^{\odot} d \mathbf{r}^{N-1} e^{-\beta U_{N}} .
$$

From the very definition of the type-B cluster

$$
p^{*}(N) \equiv \frac{1}{\Xi *} \frac{z^{N}}{N !} \int_{V} d \mathbf{r}^{N} \sum_{i=1}^{N} \delta\left(\mathbf{r}_{i}\right) e^{-\beta U_{N}},
$$

where the sum of $\delta$ functions ensures that (any) one of $N$ molecules is found at the center of $V$. The normalization conditions for $p^{* *}$ and $p^{*}$ reveal that $\Xi *=z \Xi * *$. Finally, comparison between Eqs. (19) and (33) establishes Eq. (31). In this last step, we omitted an explicit consideration of the normalization constants for the probabilities, an acceptable procedure so long as the $N=1$ term is dominant.

At this point, we clarify the nature of Reiss and Bowles's confusion manifested in Refs. 6-10. The equation they mistakenly attributed to us and criticized subsequently is given by Eq. (B5) of Ref. 10, which in the present notation may be written as

$$
p^{\otimes}(N)=\frac{z V p^{*}(N)}{p^{*}(1)+z V} . \quad[\text { Ref. 10]. }
$$

This equation differs from our Eq. (21) because $p_{c} \neq p^{\otimes}$ and $p^{\odot} \neq p^{*}$. Their argument regarding the nonuniformity of $p^{*}$ and the mapping factor related to $p^{\otimes}$ provides no grounds for challenging the validity of Eq. (21), which involves neither of these quantities.

In addition to Eq. (34), Ref. 10 mistakenly attributes the following two equations:

$$
\left\langle n_{N}\right\rangle=\frac{V_{\text {tot }}}{V} p^{\otimes}(N) \quad[\text { Ref. 10] }
$$

and

$$
\left\langle n_{N}^{*}\right\rangle=\frac{V_{\text {tot }}}{V} p_{c}(N) \quad[\text { Ref. } 10]
$$

to Refs. 1 and 3, respectively. [See Eq. (50) of Ref. 10 and the paragraph involving it.] Equation (8) in Ref. 1 is nothing 
but Eq. (18), and hence differs from Eq. (35). In fact, the former was followed by the clarification that "care is taken to avoid the surface effect," to avoid possible confusion between $p_{c}$ and $p^{\otimes}$. In Ref. 10, $\left\langle n_{N}^{*}\right\rangle$ denotes the number of $N$-sized type-B clusters in the $V_{\text {tot. }}$ This quantity does not even appear in Ref. 3. Equation (4) in Ref. 3 is equivalent to Eq. (18), and hence is different from Eq. (36).

In essence, the authors of Refs. 6-10 derived the following expression for the cluster size distribution:

$$
c(N)=\frac{1}{\theta_{N}} p^{\otimes}(N),
$$

where $\theta_{N}$ essentially is a free volume of a spherical container obtained with the center of mass of the $N$-sized cluster fixed in space. The same quantity can be understood as the free volume of the center of mass of the cluster confined to the spherical container fixed in space. The mapping factor thus deactivates the translational entropy corresponding to $V$ before activating it for $V_{\text {tot }}$. They have also shown that

$$
V \approx \theta_{N} \quad \text { and } \quad p_{c}(N) \approx p^{\otimes}(N),
$$

when the physical dimension of the cluster is negligible compared to $V$. Although we do not challenge the correctness of Eqs. (37) or (38), we also emphasize that the approximate nature of the latter by no means indicate that our Eq. (18) is an approximation. Their method and ours are simply two different ways of arriving at the same quantity, i.e., the equilibrium cluster size distribution $c$.

Though Eq. (37) remains valid outside CCL, the validity is only formal for the reasons discussed in Sec. II. Thus, the only difference is one of convenience in performing a necessary simulation. The $p_{c}$ and $p^{\odot}$ that we used yield the cluster size distribution in the simplest possible manner, while at the same time ensuring efficiency, accuracy, and robustness in the actual simulation phase.

In contrast, the method involving $p^{\otimes}$ is not advisable. First, the physical properties of the cluster evaluated by simulation reflect considerable surface effects arising from the interaction between the cluster and the system boundary. The mapping factor $V_{\text {tot }} / \theta_{N}$, in principle, removes the surface effects from the final cluster size distribution as we have just pointed out. However, the surface effect cannot be removed from other physical properties, such as the internal energy. Consequently, one cannot evaluate the entropy of a cluster directly from a single simulation using the free energy obtained from the cluster size distribution. Second, since the mapping factor involves a quantity that must be evaluated by simulation, the cluster size distribution itself is prone to any inaccuracy of simulation.

Our method avoids surface effects altogether and requires no simulation to determine the mapping factor. The trivial mapping factor $V_{\text {tot }} / V$ is rigorously correct in our construction of the distribution. The mapping factor $V_{\text {tot }} / \theta_{N}$ applies only to the method advocated by Ref. 10 and should not be used in ours.

\section{SUMMARY}

In CCL, our observational cluster method ${ }^{1,3}$ takes a particularly simple form, the validity of which is reaffirmed here. The condition of CCL was quantified and its relevance was clarified in terms of the vapor contribution as in Ref. 1. Terms that are negligible were of course neglected in formulating the method. A direct simulation by $\mathrm{Oh}$ and Zeng ${ }^{11}$ testifies to the accuracy of our method when compared to the one using CMF. Although the recent work by Reiss and Bowles ${ }^{10}$ has endorsed the high accuracy of our method, we disproved their reasoning. Most importantly, the conceptual errors they claim exist in our method result entirely from their misinterpretation of our approach and the physical or mathematical contents of partition functions involved in our method.

Construction of the equilibrium cluster size distribution indeed involves many subtle issues. Contrary to what Refs. 6-10 indicated, however, the simplicity of our method is by design and should not be regarded as an indication of our having neglected such issues.

It is regrettable that a great deal of confusion persists in the literature regarding our observational cluster method. Nonetheless, this has provided us with an opportunity to reiterate some of the subtle issues from various angles. We hope that the present paper clarifies the existing confusion and encourages further investigation of nucleation phenomena by computer simulation.

\section{ACKNOWLEDGMENTS}

This work was supported by the National Science Foundation (Grant No. CHE-9800074). One of the authors (Z.G.W.) acknowledges support from the Camille and Henry Dreyfus Foundation and a Grant by the Mobil Corporation.

\section{APPENDIX: TRANSITION MATRICES FOR GRAND CANONICAL MOVES}

To evaluate $p^{\odot}$ or $p^{*}$ by a Monte Carlo technique, the transition matrices must be designed for the grand canonical move. Thus, the distinction between $p^{\odot}$ and $p^{*}$ becomes clear by comparing such matrices for each of them. We restrict our derivation of the matrices to the grand canonical move between two microstates, one with $N$ molecules (referred to as the old configuration) and the other with $N-1$ molecules (referred to as the new configuration).

In a Monte Carlo simulation, it is advisable to ensure detailed balance

$$
\begin{aligned}
\eta_{-} & K_{-} \rho\left(N, \mathbf{r}^{N}\right) d \mathbf{r}^{N-1} \operatorname{acc}(N \rightarrow N-1) \\
& =\eta_{+} K_{+} \rho\left(N-1, \mathbf{r}^{N-1}\right) d \mathbf{r}^{N-2} \operatorname{acc}(N-1 \rightarrow N),
\end{aligned}
$$

where $\eta_{-}$is the probability of attempting a trial annihilation during one Monte Carlo cycle, $\eta_{+}$is defined similarly for a trial creation, and $\rho$ is the probability density of finding a system in a particular microstate specified by $N$ and $\mathbf{r}^{N} . K_{-}$ is the probability of proposing a particular microstate from the old one and $\operatorname{acc}(N \rightarrow N-1)$ is the probability of accepting that trial move. $K_{+}$and $\operatorname{acc}(N-1 \rightarrow N)$ refer to the corresponding quantities for the reverse move. 
In our method, the trial annihilation of a molecule was done by choosing one molecule out of $N-1$ with equal probability, since molecule 1 must be excluded, yielding

$$
K_{-}^{\odot}=\frac{1}{N-1} \frac{1}{(N-2) !},
$$

where the factorial in the denominator arises from the following consideration. The grand canonical Monte Carlo technique is aimed at evaluating multiple multidimensional integrals, each differing from the others by the value of $N$. When the simulation evolves from a state with a particular value of $N$ to $N-1$, the labeling of the molecules in the two multidimensional integrals need not be related at all, except for the label for molecule 1. Out of $(N-2)$ ! ways of labeling the molecules in the new configuration, only one such labeling is realized upon the completion of the trial move, leading to the factor in question. A similar consideration applies to the reverse move, where a creation of the $N$ th particle is attempted within a volume element $d \mathbf{r}_{N}$ taken around a point chosen with a uniform probability in the system, yielding

$$
K_{+}^{\odot}=\frac{d \mathbf{r}_{N}}{V(N-1) !} .
$$

Here, we restrict ourselves to a system of monatomic molecules for simplicity of notation. From Eq. (19)

$$
\rho\left(N ; \mathbf{r}^{N}\right)=\frac{1}{\Xi} \Xi^{\odot} \frac{z^{N}}{N !} e^{-\beta U_{N}} .
$$

Using the corresponding expression for the new configuration and restricting ourselves to an algorithm with $\eta_{-}$ $=\eta_{+}$, we see that Eq. (A1) reduces to

$$
\frac{\operatorname{acc}(N-1 \rightarrow N)}{\operatorname{acc}(N \rightarrow N-1)}=\frac{z V}{N} e^{-\beta\left(U_{N}-U_{N-1}\right)} \equiv e^{-\beta \omega^{\odot}},
$$

which has the following solution due to Metropolis: ${ }^{19}$

$$
\begin{aligned}
& \operatorname{acc}(N-1 \rightarrow N)=\min \left(1, e^{-\beta \omega^{\odot}}\right) \\
& \operatorname{acc}(N \rightarrow N-1)=\min \left(1, e^{\beta \omega^{\odot}}\right) .
\end{aligned}
$$

In a physical interpretation of the partition function, the $N$ ! factor is associated with the "sum over microstates" and should not be included in $\rho$. Otherwise, the Gibbs entropy formula would not hold. Once the partition function is written down, however, its evaluation is purely a mathematical problem and the validity of the algorithm given here is unaffected by this remark.

To validate the algorithm, we performed a direct simulation on a one-dimensional ideal gas system, for which

$$
p^{\odot}(N) \sim \frac{z^{N}}{N !} \int_{-h}^{h} d x_{2} \cdots \int_{-h}^{h} d x_{N}=\frac{z(2 h z)^{N-1}}{N !},
$$

and

$$
e^{-\beta \omega^{\odot}}=\frac{2 z h}{N}
$$

TABLE I. Comparison of $p^{\odot}$ obtained analytically and that from simulation for a one dimensional ideal gas system. $z=0.05, h=1$. Simulation 1 was performed without a bias for $10^{9} \mathrm{MC}$ steps, while simulation 2 was performed with a bias for $10^{7} \mathrm{MC}$ steps, where one MC step is defined as one trial creation or annihilation of a molecule.

\begin{tabular}{llll}
\hline \hline $\mathrm{N}$ & Analytical & Simulation 1 & Simulation 2 \\
\hline 1 & 0.9508 & 0.9508 & 0.9508 \\
2 & $0.4754 \times 10^{-1}$ & $0.4755 \times 10^{-1}$ & $0.4757 \times 10^{-1}$ \\
3 & $0.1585 \times 10^{-2}$ & $0.1582 \times 10^{-2}$ & $0.1585 \times 10^{-2}$ \\
4 & $0.3962 \times 10^{-4}$ & $0.4015 \times 10^{-4}$ & $0.3962 \times 10^{-4}$ \\
5 & $0.7924 \times 10^{-6}$ & $0.8150 \times 10^{-6}$ & $0.7935 \times 10^{-6}$ \\
6 & $0.1321 \times 10^{-7}$ & $0.1800 \times 10^{-7}$ & $0.1324 \times 10^{-7}$ \\
\hline \hline
\end{tabular}

Table I compares $p^{\odot}$ obtained analytically and that from a simulation, where the distribution is truncated at $N=6$. In the first simulation, no bias was employed to enhance the sampling of less probable states. As a result, the agreement worsens with increasing $N$ since larger $N$ values have smaller probability and hence lead to larger statistical errors. When a bias is introduced, the agreement improved dramatically. In any event, the agreement is excellent for all $N$ values considered, thereby demonstrating the validity of the algorithm.

In the present context, $p^{*}$ may be understood as the distribution determined by a simulation protocol that is aimed at sampling from $p^{\odot}$ but designed by following the interpretation of $p^{\odot}$ as given in Ref. 10. The algorithm for $p^{*}$ differs from the one just described in that the relabeling of molecules upon completion of a grand canonical Monte Carlo move must include molecule 1 as well. On the other hand, the molecule that is at the center of the system must not be removed. Thus, in place of $K_{-}^{\odot}$ and $K_{+}^{\odot}$ given above, we have

$$
K_{-}^{*}=\frac{1}{N-1} \frac{1}{(N-1) !} \quad \text { and } \quad K_{+}^{*}=\frac{d \mathbf{r}_{N}}{V N !} .
$$

Since $\rho$ is unchanged, the transition matrix is given by Eq. (A6) with $\omega^{*}$ replacing the $\omega^{\odot}$, where

$$
e^{-\beta \omega^{*}} \equiv \frac{z V}{N-1} e^{-\beta\left(U_{N}-U_{N-1}\right)}
$$

Insofar as $\omega^{\odot} \neq \omega^{*}$, we may expect that $p^{\odot} \neq p^{*}$. In fact, $p^{*}$ can be shown to be identical to $p^{* *}$ given by Eq. (32). Following a similar procedure that leads to $\omega^{\odot}$ with a proper modification to $\rho$, one finds that the transition matrices that sample from $p^{* *}$ are given by Eq. (A6) but with $\omega^{\odot}$ replaced by $\omega^{*}$. Since the factors arising from the relabeling of molecules do not enter explicitly in the implementation of a simulation, we see that the simulation protocol for $p^{* *}$ is identical to that for $p^{*}$. Noting that the range of $N$ is the same for $p^{*}$ and $p^{* *}$, we see that $p^{*}=p^{* *}$. Comparison between Eqs. (19) and (32) leads to $p^{\odot}=p^{* * / N}$, provided that $N=1$ dominates these probabilities. Thus, we reverified Eq. (31). 
${ }^{1}$ I. Kusaka, Z.-G. Wang, and J. H. Seinfeld, J. Chem. Phys. 108, 3416 (1998).

${ }^{2}$ I. Kusaka and D. W. Oxtoby, J. Chem. Phys. 110, 5249 (1999).

${ }^{3}$ I. Kusaka, D. W. Oxtoby, and Z.-G. Wang, J. Chem. Phys. 111, 9958 (1999).

${ }^{4}$ I. Kusaka and D. W. Oxtoby, J. Chem. Phys. 111, 1104 (1999).

${ }^{5}$ I. Kusaka and D. W. Oxtoby, J. Chem. Phys. 115, 4883 (2001).

${ }^{6}$ H. Reiss, J. Mol. Struct. 485-486, 465 (1999).

${ }^{7}$ H. Reiss and R. Bowles, J. Chem. Phys. 111, 9965 (1999).

${ }^{8}$ R. K. Bowles, J. Chem. Phys. 112, 1122 (2000).

${ }^{9}$ H. Reiss and R. K. Bowles, J. Chem. Phys. 112, 1390 (2000).

${ }^{10}$ H. Reiss and R. K. Bowles, J. Chem. Phys. 113, 8615 (2000).

${ }^{11}$ K. J. Oh and X. C. Zeng, J. Chem. Phys. 114, 2681 (2001).
${ }^{12}$ J. K. Lee, J. A. Barker, and F. F. Abraham, J. Chem. Phys. 58, 3166 (1973).

${ }^{13}$ F. H. Stillinger, Jr., J. Chem. Phys. 38, 1486 (1963).

${ }^{14}$ W. J. Dunning, in Nucleation, edited by A. C. Zettlemoyer (Dekker, New York, 1969), pp. 1-67.

${ }^{15}$ K. Nishioka and G. M. Pound, Adv. Colloid Interface Sci. 7, 205 (1977).

${ }^{16}$ J. W. Gibbs, Elementary Principles in Statistical Mechanics, (Ox Bow, Woodbridge, CT, 1981).

${ }^{17}$ J. W. Gibbs, The Scientific Papers of J. Willard Gibbs (Ox Bow, Woodbridge, CT, 1993), Vol. I, Thermodynamics.

${ }^{18}$ D. W. Oxtoby and R. Evans, J. Chem. Phys. 89, 7521 (1988).

${ }^{19}$ N. Metropolis, A. W. Rosenbluth, M. N. Rosenbluth, A. H. Teller, and E. Teller, J. Chem. Phys. 21, 1087 (1953) 